\title{
Bosonization Method for Second Super Quantization
}

Alexander Dynin

To cite this article: Alexander Dynin (2010) Bosonization Method for Second Super Quantization, Journal of Nonlinear Mathematical Physics 17:Supplement 1, 1-13, DOI: https://doi.org/10.1142/S1402925110000763

To link to this article: https://doi.org/10.1142/S1402925110000763

Published online: 04 January 2021 


\title{
BOSONIZATION METHOD FOR SECOND SUPER QUANTIZATION
}

\author{
ALEXANDER DYNIN \\ Department of Mathematics \\ Ohio State University Columbus, OH 43210, USA \\ dynin@math.ohio-state.edu \\ Received 1 July 2009 \\ Revised 10 August 2009 \\ Accepted 20 September 2009
}

In memoriam of F. A. Berezin (1931-1980)

\begin{abstract}
A boson-fermion correspondence allows an analytic definition of functional super derivative, in particular, and a bosonic functional calculus, in general, on Bargmann Gelfand triples for the second super quantization. A Feynman integral for the super transformation matrix elements in terms of bosonic anti-normal Berezin-Sudarshan symbols is rigorously constructed.

Keywords: Second super quantization; functional method in quantum field theory; anti-normal quantization; anti-Wick quantization; Berezin quantization.

Mathematics Subject Classification: 81S05, $81 \mathrm{~S} 10$ (Primary) 81S30, 81T08, $81 \mathrm{~T} 60$ (Secondary)
\end{abstract}

\section{Introduction}

\subsection{Preview}

We begin with a summary of research directions opened by Berezin's monograph "Method of second quantization" [6]: one is a super second quantization, and another is an extension of quantum mechanical Schrödinger picture to quantum field theory.

Extending KREE's second quantization [17] and Hida's white noise calculus (see, e.g., [26]) we develop the second quantization in super Bargmann-Fock Gelfand triples to account for the quantum states knocked out by a violent Schrödinger operator (see below the quote from Dirac).

The "violence" of a Schrödinger operator means that the domain of the operator is not dense in the Fock space. Actually, it is continuous operator from a nuclear Frechet space of test functionals of classical fields to the anti-dual space of functional of distributions. (Apparitions of Gelfand triples are seen in [4, Subsecs. II.2.5 and II.3.4].) 
The related $c h$ difficulty in relativistic quantum field theory was discovered by [19] in 1931: boundedness of velocities by the light velocity $c$ implies boundedness of momenta, so that, by the uncertainty principle, exact values of a quantized field do not exist. However the difficulty is resolved in Gelfand triples via canonical commutation relations between creation operators of test functionals and annihilation operators of distribution functionals.

Following [11], Schrödinger super equations in a Gelfand triple is solved via mathematically rigorous anti-normal Feynman super integral.

\subsection{Berezin's legacy}

In 1956, I. M. Gelfand initiated F. Berezin into Quantum Field theory. Berezin was greatly influenced by K. Friedrichs' dictum:

According to Niels Bohr, any attempt at a sharp definition of physical concepts must even violate their real physical meaning. Therefore, the mathematician's desire for a deductive presentation of physical theory cannot be established in principle. On the other hand, it seems justified to strive for a precise definition of the intrinsic mathematical meaning of mathematical notions employed in [...] quantum theory [14].

Berezin's goal was

to construct a noncontradictory quantum field theory. Without exaggeration, it can be said that he regarded almost all of his work (on the $N$ particle problem, quantization, superanalysis) as stepping stones to this difficult problem [22].

In this respect Berezin's monograph [4] became the next landmark after [14]. It develops the Fock method of generating functionals of bosonic states into a correspondence between quantum operators and their normal functional symbols in the framework of (anti-)holomorphic Fock spaces of bosonic states and, for the first time, of fermionic states. The normal representation of bounded operators in Fock spaces came as a surprise and, certainly, asked for further generalizations. That was done in bosonic Gelfand triples of quantum field theory [17] and of white noise calculus (see [26]). For fermions, this is done in this paper.

The follow up Berezin's papers on quantization with finite number of freedom degrees have been stepping stones toward quantum field theory. In particular, analytical possibilities of anti-normal (aka Berezin, Hisumi, Sudarshan, diagonal) symbols have been explored in [5].

Parallelism between bosonic and fermionic Fock spaces was already discussed in [12]. In the bosonic case the functional method of the second quantization was proposed in the sequel [13]. The "striking similarity" of analysis of bosonic and fermionic generating functionals [4, Introduction] inspired Berezin's fermionic extension of the functional method. (By penetrating remark in [24, Sec. I.5], for this it was necessary to understand well both bosonic and fermionic cases. Otherwise they are not so similar....) Eventually, this led to super analysis (cp. [6]) and to new beginnings in theory of infinite linear groups (cp. [24]). 
By $[4$, Introduction],

[...] functionals may be imagined, roughly speaking, as functions of infinitely many variables. In usual quantum mechanics, the number of variables of functions representing the state space is the number of freedom degrees.

Thus there arises an interpretation of the second quantization problems as quantum mechanics problems with infinitely many degrees of freedom and a natural desire to approximate these problems via problems with finite, but large, number of degrees of freedom.

In the last section of this paper we use such interpretation to derive a rigorous antinormal Feynman integral for the matrix elements Schrödinger operators in super Gelfand triples.

\subsection{Quantum mechanics vs quantum field theory}

Quantum Mechanics was invented by W. Heisenberg in 1925. The famous monographs [25] and [29] summarized corresponding new mathematics. The main goal was to comprehend canonical commutation relations and ensuing non-commutativity of quantum variables of Heisenberg's and E. Schrödinger's Quantum Mechanics.

Von Neumann defined and named Hilbert spaces to honor Hilbert theory of quadratic forms. He replaced the latter by (unbounded) self-adjoint operators corresponding to quantum observables. Weyl quantization converts classical observables into operators. A generalized quantization rule was proposed by [30] and the corresponding formal calculus was developed by [1].

The fundamental quantum uncertainty principle has the mathematical underpinning of canonical commutation relations. Weyl conjectured and von Neumann proved the unitary equivalence of irreducible unitary representations of bosonic canonical commutation relations with a finite number of degrees of freedom. The corresponding theorem for fermionic canonical commutation relations was established in 1927 by P. Jordan and E. Wigner.

In contrast to quantum Mechanics, mathematics of quantum Field Theory has been developing much slower, mainly because of the infinite number of degrees of freedom. There was vivid correspondence between W. Heisenberg, P. Jordan and W. Pauli about possibilities of Volterra functional calculus.

There is no uniqueness theorem for unitary representations of the canonical commutation relations (cp. the monograph [14]) (it is presumed that this non-uniqueness was discovered by von Neumann in late 1930's). However, under an additional requirement of existence of the fiducial quantum vacuum state, they are unitarily equivalent.

Friedrichs' monograph [14] was an attempt to do for Quantum Field Theory what von Neumann had done for Quantum Mechanics. Unfortunately, it lacked von Neumann's elegance. F. Berezin's use of holomorphic Fock-Bargmann representations in his monograph [4] clarifies the subject considerably.

In 1927, [9] introduced the method of second quantization in Quantum Electrodynamics as a system of harmonic oscillators. 
In 1931, [18] proposed an alternative configuration space quantization method diagonalizing the field multiplication operators. (Monograph [4] starts with such configuration space, no attribution already needed.)

The foundational paper [12] on Fock representation of the bosonic and fermionic canonical commutation relations with the infinite number of degrees of freedom begins as follows (in translation)

The fact that the second quantization method is equivalent to the method of usual wave functions on a configuration space is known in principle. In this paper this is traced in detail.

In the presence of a unique vacuum vector the representations of canonical commutation relations are unique up to unitary equivalence.

The sequel [13] introduced the method of generating functionals for bosons. Both Fock's papers are formal calculations.

In the beginning, W. Heisenberg, P. Jordan and W. Pauli had idea to use the canonical commutation relations to extend Heisenberg and Schrödinger pictures of quantum mechanics to quantum field theory. There was a vivid discussion of "Volterra mathematics" in their correspondence.

However, according to P. Dirac [10], Section "Relationship of the Heisenberg and Schrödinger Pictures",

The interactions that are physically important in quantum field theory are so violent that they will knock any Schrödinger state vector out of Hilbert space in the shortest possible time interval.

[...] It is better to abandon all attempts at using the Schrödinger picture with these Hamiltonians.

[...] I don't want to assert that the Schrödinger picture will not come back. In fact, there are so many beautiful things about it that I have the feeling in the back of my mind that it ought to come back. I am really loath to have to give it up.

In this paper, the Schrödinger picture has been resurrected in the framework of Gelfand triples, cp. [15].

\section{Bosonic Gelfand Triples}

\subsection{Holomorphic states}

In this section, $\mathcal{H}$ denotes an (infinite dimensional) bosonic complex separable Hilbert *-space with conjugation (cp. [4]).

Sandwich $\mathcal{H}$ into a Gelfand nuclear $*$-triple (cp. [15])

$$
\mathcal{H}^{\infty} \subset \mathcal{H} \subset \mathcal{H}^{-\infty}
$$

where $\mathcal{H}^{\infty}$ is a nuclear countably Hilbert $*$-space, $\mathcal{H}^{-\infty}$ is its topological $*$-dual with respect to the Hermitian product on $\mathcal{H}$. The imbeddings are continuous with dense ranges and real, i.e., commute with the conjugation. 
By Minlos' theorem, space $\mathcal{H}^{-\infty}$ carries the probability Gauss-Radon measure $d z^{*} d z e^{-z^{*} z}$. This symbolic expression is meaningful as a cylindrical measure on $\mathcal{H}^{-\infty}$ which extends to the Gauss-Radon measure. We use the same notation for both measures because it allows integration by parts and Fubini theorem which hold for integrals of cylindrical functions followed by limit transition to the wider class of integrable functions.

Fernique's theorem (see [7, Chapter 2]) implies that there exists a positive constant $c$ such that if a functional $\Psi\left(z^{*}\right)$ on $\mathcal{H}^{-\infty}$ is continuous and $\Psi \prec e^{-c z^{*} z}$, then $\Psi\left(z^{*}\right)$ is integrable on $\mathcal{H}$.

The Bargmann space (see, e.g., [4, Chapter I]) is the (complete) complex Hilbert space of Gâteaux entire functionals $\Psi=\Psi\left(z^{*}\right)$ on $\mathcal{H}^{-\infty}$ with conjugation

$$
\Psi^{*}=\Psi^{*}(z) \equiv \overline{\Psi\left(z^{*}\right)}
$$

and integrable Hermitian sesqui-linear inner product

$$
\Psi^{*} \Phi \equiv \int d z^{*} d z e^{-z^{*} z} \Psi^{*}(z) \Phi\left(z^{*}\right)
$$

The integral is denoted also as the Gaussian contraction $\Psi^{*}(z) \Phi\left(z^{*}\right)$.

The exponential functionals

$$
e^{z}\left(z^{*}\right) \equiv e^{z^{*} z}, \quad z \in \mathcal{H}^{\infty}
$$

belong to $\mathcal{B}^{0}$ since $e^{z^{*}} e^{z}=e^{z^{*} z}<\infty$. Indeed

$$
e^{z^{*}} e^{\xi}=\int d z^{*} d z e^{-z^{*} z} e^{z^{*} z+z^{*} \xi}=e^{z^{*} \xi} \int d z^{*} d z e^{-\left(z^{*}-z^{*}\right)(z-\xi)}=e^{z^{*} \xi}
$$

They form a continual orthogonal basis of exponential functionals in $\mathcal{B}^{0}$ (see, e.g., [4, Chapter I]): If $\Psi=\Psi\left(z^{*}\right) \in \mathcal{B}^{0}$, then the Borel transform

$$
\Psi\left(z^{*}\right)=e^{-z^{*} z} \int d \zeta d \zeta^{*} e^{-\zeta^{*} \zeta} \widetilde{\Psi}(\zeta) e^{z^{*} \zeta}, \quad \widetilde{\Psi}(\zeta) \equiv \Psi^{*} e^{\zeta}
$$

is a unitary operator in $\mathcal{B}^{0}$.

The orthogonal basis is overcomplete since

$$
e^{z}=\int d \zeta d \zeta^{*} e^{-\zeta^{*} \zeta} e^{z^{*} \zeta}
$$

The Bargmann-Hida space $\mathcal{B}^{\infty}$ is the vector space of of Gâteaux entire test functionals $\Psi\left(z^{*}\right)$ on $\mathcal{H}^{-\infty}$ of the (topological) second order and minimal type, i.e., for any $s \geq 0$ and $\epsilon>0$, there exists a constant $C>0$ such that

$$
\left|\Psi\left(z^{*}\right)\right| \leq C e^{\epsilon\left\|z^{*}\right\|_{-s}^{2}}, \quad z^{*} \in \mathcal{H}^{-s} .
$$

$\mathcal{B}^{\infty}$ is a nuclear space of type $(\mathrm{F}),{ }^{\mathrm{a}}$ dense in $\mathcal{B}^{0}$ (see, e.g., [26, Subsec. 3.6]).

\footnotetext{
${ }^{\mathrm{a}}(\mathrm{F})$ is for locally convex Frechet spaces and (DF) is for their topological dual. The spaces under consideration are nuclear countably Hilbert spaces and their topological dual and the main thing here is of course the Grothendieck-Schwartz kernel theorem, see, e.g., [27].
} 
Actually, the topology of $\mathcal{B}^{\infty}$ is defined by the norms

$$
\|\Psi\|_{s, \epsilon} \equiv \sup _{z^{*}}\left|\Psi\left(z^{*}\right)\right| e^{-\epsilon\left\|z^{*}\right\|_{-s}^{2}} .
$$

Again, by [26, Subsec. 3.6], Borel transform is a topological automorphism of $\mathcal{B}^{\infty}$.

Bargmann-Hida space $\mathcal{B}^{-\infty}$ of generalized functionals $\Psi^{*}(z)$ on $\mathcal{H}^{\infty}$ is the strong antidual space of $\mathcal{B}^{\infty}$ (and therefore, of type $(\mathrm{DF})$ ). The Borel transform $\widetilde{\Psi}^{*}(z)$ of $\mathcal{B}^{-\infty}$ is defined as the anti-dual of the Borel transform of $\mathcal{B}^{\infty}$ of $\mathcal{B}^{-\infty}$ (and therefore, a topological automorphism).

By, e.g., [26, Subsec. 3.6], the generalized functionals are characterized as entire functionals of the (bornological) second order on $\mathcal{H}^{\infty}$, i.e., there exist positive constants $C, K$ and $s \geq 0$ such that

$$
|\Psi(z)| \leq C e^{K\|z\|_{s}^{2}}, \quad z \in \mathcal{H}^{s} .
$$

We get the Bargmann-Hida Gelfand triple of holomorphic states

$$
\mathcal{B}^{\infty} \subset \mathcal{B}^{0} \subset \mathcal{B}^{-\infty} \text {. }
$$

The vector spaces $\mathcal{B}^{\infty}$ and $\mathcal{B}^{-\infty}$ are locally convex commutative topological algebras with the point-wise multiplication. Moreover, we have Taylor series expansions

$$
\begin{gathered}
\Psi\left(z^{*}+w^{*}\right)=\sum_{n=0}^{\infty} \frac{\partial_{z^{*}}^{n} \Psi\left(z^{*}\right)}{n !} w^{* n} \quad \text { for } \Psi \in \mathcal{B}^{\infty} \\
\Psi(z+w)=\sum_{n=0}^{\infty} \frac{\partial_{z}^{n} \Psi(z)}{n !} w^{n} \quad \text { for } \Psi \in \mathcal{B}^{-\infty}
\end{gathered}
$$

By conjugating $z$ to $z^{*}$, we convert $\mathcal{H}^{\infty} \subset \mathcal{H}^{0} \subset \mathcal{H}^{-\infty}$ into the conjugate Gelfand triple $H^{* \infty} \subset H^{* 0} \subset H^{*-\infty}$. Their direct product

$$
\mathcal{H}^{\infty} \times H^{* \infty} \subset \mathcal{H}^{0} \times H^{* 0} \subset H^{-\infty} \times \mathcal{H}^{*-\infty}
$$

carries the complex conjugation $\left(z, w^{*}\right)^{*} \equiv\left(w, z^{*}\right)$.

The Bargmann-Hida Gelfand triple associated with (2.14) is

$$
\left(\mathcal{B} \otimes \mathcal{B}^{*}\right)^{\infty} \subset\left(\mathcal{B} \otimes \mathcal{B}^{*}\right)^{0} \subset\left(\mathcal{B} \otimes \mathcal{B}^{*}\right)^{-\infty} .
$$

Entire functionals $\Theta\left(z, w^{*}\right) \in\left(\mathcal{B} \otimes \mathcal{B}^{*}\right)^{-\infty}$ are uniquely defined by their restrictions $\Theta\left(z, z^{*}\right)$ to the real diagonal. If $\overline{\Theta\left(z, z^{*}\right)}=\Theta\left(z, z^{*}\right)$, then $\Theta\left(z, z^{*}\right)$ is a classical observable on the phase space $\mathcal{H}^{\infty}$.

By Bargmann-Segal transform (see, e.g., [26, S-transform]), Cook-Fock Gelfand *-triple $\mathbf{F}$ is unitarily equivalent to Bargmann-Hida Gelfand $*$-triple $\mathbf{B}$.

\subsection{Second quantization of classical bosonic observables}

For $z \in \mathcal{H}^{\infty}, z^{*} \in \overline{\mathbb{C}} \mathcal{H}_{0}^{-\infty}$, define four continuous operators of multiplication and directional complex differentiation (operators of creation and annihilation):

$$
\begin{gathered}
\hat{z}: \mathcal{B}^{\infty} \rightarrow \mathcal{B}^{\infty}, \quad \hat{z} \Psi\left(\zeta^{*}\right) \equiv z \Psi\left(\zeta^{*}\right)=\left(\zeta^{*} z\right) \Psi\left(\zeta^{*}\right) \\
\hat{z}^{\dagger}: \mathcal{B}^{-\infty} \rightarrow \mathcal{B}^{-\infty}, \quad \hat{z}^{\dagger} \Psi(\zeta) \equiv \partial_{z} \Psi(\zeta) ;
\end{gathered}
$$




$$
\begin{gathered}
\widehat{z^{*}}: \mathcal{B}^{-\infty} \rightarrow \mathcal{B}^{-\infty}, \quad \widehat{z^{*}} \Psi(\zeta) \equiv z^{*} \Psi(\zeta)=\left(z^{*} \zeta\right) \Psi(\zeta) \\
{\widehat{z^{*}}}^{\dagger}: \mathcal{B}^{\infty} \rightarrow \mathcal{B}^{\infty}, \quad{\widehat{z^{*}}}^{\dagger} \Psi\left(\zeta^{*}\right) \equiv \partial_{z^{*}} \Psi\left(\zeta^{*}\right)
\end{gathered}
$$

The continuity of multiplications is straightforward; the continuity of directional differentiations follows from Cauchy integral formula for the derivative of a holomorphic function.

These operators generate strongly continuous Abelian operator groups in $\mathcal{B}^{\infty}$ and $\mathcal{B}^{-\infty}$ :

$$
\begin{aligned}
& e^{\hat{z}}: \mathcal{B}^{\infty} \rightarrow \mathcal{B}^{\infty}, \quad e^{\hat{z}} \Psi\left(\zeta^{*}\right)=e^{\zeta^{*} z} \Psi\left(\zeta^{*}\right) ; \\
& e^{\hat{z}^{\dagger}}: \mathcal{B}^{-\infty} \rightarrow \mathcal{B}^{-\infty}, \quad e^{\hat{z}^{\dagger}} \Psi(\zeta)=\Psi(\zeta+z) ; \\
& e^{\widehat{z^{*}}}: \mathcal{B}^{-\infty} \rightarrow \mathcal{B}^{-\infty}, \quad e^{\widehat{z^{*}}} \Psi(\zeta)=e^{z^{*} \zeta} \Psi(\zeta) ;
\end{aligned}
$$

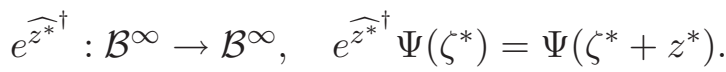

The only nontrivial commutation relations for the groups

$$
\left[e^{\widehat{z^{*}}}, e^{\hat{z}}\right]=e^{z^{*} z}, \quad\left[e^{\hat{z}^{\dagger}}, e^{\widehat{z^{*}}}\right]=e^{z z^{*}}
$$

imply the only nontrivial commutation relations of their generators

$$
\left[{\widehat{z^{*}}}^{\dagger}, \hat{z}\right]=z^{*} z, \quad\left[\hat{z}^{\dagger}, \widehat{z}^{*}\right]=z z^{*}
$$

The normal quantization $\Theta\left(\hat{z},{\widehat{z^{*}}}^{\dagger}\right)$ of $\Theta\left(z, z^{*}\right) \in\left(\mathcal{B} \otimes \mathcal{B}^{*}\right)^{-\infty}$ is defined as the continuous linear operator

$$
\Theta\left(\hat{z},{\widehat{z^{*}}}^{\dagger}\right): \mathcal{B}^{\infty} \rightarrow \mathcal{B}^{-\infty}
$$

via the sesqui-linear quadratic form (in Einstein-DeWitt contraction notation, i.e., in the integral contraction over conjugated continual variables the integration symbols are skipped)

$$
\Psi^{*}(z) \Theta\left(\hat{z},{\widehat{z^{*}}}^{\dagger}\right) \Psi\left(z^{*}\right) \equiv \widetilde{\Theta}\left(\zeta^{*}, \zeta\right) e^{\hat{z}} e^{\widehat{z}^{\dagger}} \widetilde{\Psi}^{*}(\zeta) \widetilde{\Psi}\left(\zeta^{*}\right)
$$

The sesqui-holomorphic $\widetilde{\Theta}\left(\zeta^{*}, \eta\right)$ is the normal symbol of the operator $\Theta\left(\hat{z}, \widehat{z^{*}}\right)$ uniquely defined by its restriction $\widetilde{\Theta}\left(\zeta^{*}, \zeta\right)$ to the real diagonal.

Similarly, the kernel $K\left(z, y^{*}\right)$ of the operator $\Theta\left(\hat{z}, \widehat{z^{*}}\right)$ is uniquely defined by its diagonal restriction

$$
\begin{gathered}
K\left(z, z^{*}\right) \equiv e^{\zeta^{*}}(z) \Theta\left(\hat{z},{\widehat{z^{*}}}^{\dagger}\right) e^{\zeta}\left(z^{*}\right) \\
\stackrel{(2.27)}{=} \widetilde{\Theta}\left(\zeta^{*}, \zeta\right) e^{\hat{z}} e^{{\widehat{z^{*}}}^{\dagger}} e^{z}\left(\zeta^{*}\right) e^{z^{*}(\zeta)} \stackrel{(2.16),(2.19))}{=} \Theta\left(z, z^{*}\right) e^{z z^{*}} \in\left(\mathcal{B} \otimes \mathcal{B}^{*}\right)^{-\infty} .
\end{gathered}
$$

Thus the correspondence between quantum observables $\Theta\left(\hat{z}, \widehat{z^{*}}\right)$ and classical observables $\Theta\left(z, z^{*}\right)$ is one-to-one:

$$
K\left(z, z^{*}\right)=\Theta\left(z, z^{*}\right) e^{z z^{*}}
$$

Since $e^{z z^{*}}$ is the integral kernel of the orthogonal projection of $\left(\mathcal{B} \otimes \mathcal{B}^{*}\right)^{-\infty}$ onto $\mathcal{B}^{* \infty}$, the classical variable $\Theta\left(z^{*}, z\right)$ is the Berezin (aka anti-normal, diagonal, or Sudarshan) symbol 
of the operator $\Theta\left(\hat{z},{\widehat{z^{*}}}^{\dagger}\right)$, i.e., the compression of the multiplication with $\Theta\left(z^{*}, z\right)$ to $\mathcal{B}^{* \infty}$ :

$$
\Theta\left(\hat{z},{\widehat{z^{*}}}^{\dagger}\right) \Psi\left(z^{*}\right)=e^{z^{*} z} \Theta\left(z^{*}, z\right) \Psi^{*}(z) .
$$

The symbol is called anti-normal because

$$
\Psi^{*}(z) e^{z z^{*}} \Theta\left(z^{*}, z\right) \Psi\left(z^{*}\right) \stackrel{(2.24)}{=} \widetilde{\Psi}^{*}\left(\zeta^{*}\right) \widetilde{\Theta}\left(\zeta^{*}, \zeta\right) e^{{\widehat{z^{*}}}^{\dagger}} e^{\hat{z}} \widetilde{\Psi}(\zeta)
$$

Compare with the (opposite) normal operator ordering in (2.27).

For $\Theta \in\left(\mathcal{B} \otimes \mathcal{B}^{*}\right)^{\infty}$, we have, by Taylor expansion and integration by parts,

$$
\begin{aligned}
& \Theta\left(z, z^{*}\right)=e^{-z^{*} z} \tilde{\Theta}\left(\zeta^{*}, \zeta\right) e^{\zeta^{*} z} e^{z^{*} \zeta} \\
& =\int d \zeta^{*} d \zeta \tilde{\Theta}\left(\zeta^{*}, \zeta\right) e^{-\left(z^{*}-\zeta^{*}\right)(z-\zeta)} \\
& =\int d \zeta^{*} d \zeta e^{-\zeta^{*} \zeta} \tilde{\Theta}\left(z^{*}-\zeta^{*}, z-\zeta\right) \\
& =\sum_{k, m} \frac{(-1)^{k+m}}{k m} \int d \zeta^{*} d \zeta e^{-\zeta^{*} \zeta} \partial_{\zeta^{*}}^{k} \partial_{\zeta}^{m} \tilde{\Theta}\left(z^{*}, z\right)\left(\zeta^{* k} \zeta^{m}\right) \\
& =\sum_{k} \frac{1}{k} \partial_{\zeta^{*}}^{k} \partial_{\zeta}^{m} \tilde{\Theta}\left(z^{*}, z\right) \int d \zeta^{*} d \zeta e^{-\zeta^{*} \zeta}\left(\zeta^{* k} \zeta^{m}\right) \\
& =e^{\left(\partial_{\zeta^{*}} \partial_{\zeta}\right) / 2} \tilde{\Theta}\left(z^{*}, z\right),
\end{aligned}
$$

since $\widehat{\zeta}^{\dagger}=\partial_{\zeta}$. (Note: The contraction $\partial_{\zeta^{*}} \partial_{\zeta}$ is an infinite dimensional complex Laplacian.)

Since $\left(\mathcal{B} \otimes \mathcal{B}^{*}\right)^{\infty}$ is dense in $\left(\mathcal{B} \otimes \mathcal{B}^{*}\right)^{-\infty}$, we get a relationship between the normal and anti-normal symbols for all $\Theta \in\left(\mathcal{B} \otimes \mathcal{B}^{*}\right)^{-\infty}$ as

$$
\Theta\left(z^{*}, z\right)=e^{\left(\partial_{\zeta^{*}} \partial_{\zeta}\right) / 2} \tilde{\Theta}\left(z^{*}, z\right) \text { and } \tilde{\Theta}\left(z^{*}, z\right)=e^{-\left(\partial_{\zeta^{*}} \partial_{\zeta}\right) / 2} \Theta\left(z, z^{*}\right) .
$$

E.g., the constant functional 1 is both the normal and anti-normal symbol of the identity operator; the normal symbol of the number operator is the functional $z^{*} z$, and its antinormal symbol is $z^{*} z-\frac{1}{2}$.

\section{Gelfand Super Triples}

\subsection{Bosonization of Gelfand super triples}

Consider an infinite dimensional (separable) complex super Hilbert $*$-space $\mathcal{H}$, i.e., a $\mathbb{Z} / 2$ graded space

$$
\mathcal{H}=\mathcal{H}_{0} \oplus \mathcal{H}_{1}, \quad \operatorname{dim}\left(\mathcal{H}_{1}\right)=\infty
$$

of elements $z=z_{0} \oplus z_{1}$ with bosonic even parts $z_{0}$ and the fermionic odd parts $z_{1}$. We denote the parity of a homogeneous element $z$ by $p(z)$. Furthermore, $\mathcal{H}$ is endowed with an anti-linear even involution $*: z \mapsto z^{*}$, i.e.,

$$
(c z)^{*}=\bar{c} z^{*}, \quad c \in \mathbb{C}, \quad z^{* *}=z .
$$


The super Hermitian product $z^{*} w$ on $\mathcal{H}$ is a super sesqui-linear form on $\mathcal{H}$ such that for homogeneous $z, w \in \mathcal{H}$

$$
\overline{z^{*} w}=(-1)^{p(z) p(w)} w^{*} z, \quad z_{0}^{*} z_{0} \geq 0, \quad-i z_{1}^{*} z_{1} \geq 0 .
$$

In particular, the super Hermitian quadratic form $z_{1}^{*} z_{1}$ is purely imaginary with $\operatorname{Im}\left(z_{1}^{*} z_{1}\right) \geq$ 0. Moreover, $\mathcal{H}_{0}$ and $\mathcal{H}_{1}$ are orthogonal superspaces.

Infinite dimensional separable Hilbert *-spaces haze real orthonormal bases. Therefore, they are $*$-unitarily isomorphic, i.e., the equivalence unitary operator commutes with complex conjugations (cp. [4, Introduction]).

The infinite dimensional separable super Hilbert $*$-space $\mathcal{H}_{1}$ is super unitary equivalent to the Hilbert $*$-space $\mathcal{L}^{2}(\mathbf{R})$ of complex-valued functions on the real line with the usual complex conjugation.

Then the anti-symmetric Hilbert tensor power $\otimes_{1}^{n} \mathcal{H}_{1}$ is $*$-unitarily equivalent to the Hilbert $*$-subspace of anti-symmetric functions $f\left(x_{1}, \ldots, x_{n}\right)$ in $\mathcal{L}^{2}\left(\mathbf{R}^{n}\right)$.

Both symmetric and anti-symmetric functions on $\mathbf{R}^{n}$ are uniquely defined by restriction to the open subset of $\mathbf{R}^{n}$

$$
\check{\mathbf{R}}^{n} \equiv\left\{\check{x}=\left(x_{1}, x_{2}, \ldots, x_{n}\right) \mid x_{1}<x_{2}<\cdots<x_{n}\right\} .
$$

The super symmetrization $S_{0}^{n}$ of $f(\check{x})$ on $\check{\mathbf{R}}^{n}$ produces a unique supersymmetric function $S_{0}^{n}(f)\left(x_{1}, \ldots, x_{n}\right)$ almost everywhere on $\mathbf{R}^{n}$. Furthermore, $S_{0}^{n}$ generates a $*$-unitary operator from $\otimes_{1}^{n} \mathcal{H}_{1}$ onto $\otimes_{0}^{n} \mathcal{H}_{1}$ (cp., [21, pp. 59-60]). This implies *-bosonization unitary isomorphisms

$$
\varpi^{m, n}: \otimes_{0}^{m} \mathcal{H}_{0} \oplus \otimes_{1}^{n} \mathcal{H}_{1} \rightarrow \otimes_{0}^{m} \mathcal{H}_{0} \oplus \otimes_{0}^{n} \mathcal{H}_{1}
$$

The direct sum $\varpi=\oplus_{m, n} \varpi^{m, n}$ is the $*$-unitary bosonization of the super Fock space $\mathcal{F}(\mathcal{H})=\mathcal{H}_{0} \otimes \mathcal{H}_{1}$. It converts $\mathcal{F}(\mathcal{H})$ into the bosonic Fock space $\mathcal{F}\left(\mathcal{H}_{\infty}\right)$ over the bosonic Hilbert $*$-space $H_{1}=\mathcal{H}_{0} \oplus \varpi^{0,1} \mathcal{H}_{1}$. The odd mapping $\varpi$ is linear and super unitary: $\varpi^{\dagger}=\varpi^{-1}$.

Let $\mathcal{H}_{1}$ be sandwiched into the bosonic Bargmann-Hida Gelfand *-triple

$$
\mathcal{H}_{1}^{\infty} \subset \mathcal{H}_{1} \subset \mathcal{H}_{1}^{-\infty}
$$

Then $\mathcal{H}$ is sandwiched into the Gelfand super $*$-triple

$$
\mathcal{H}^{\infty} \subset \mathcal{H} \subset \mathcal{H}^{-\infty}
$$

where $\mathcal{H}^{\infty} \equiv \varpi^{-1}\left(\mathcal{H}_{1}^{\infty}\right)$ is a countably super Hilbert $*$-space, and $\mathcal{H}^{-\infty}$ is its topological *-dual.

As a consequence, the corresponding holomorphic Bargmann-Hida Gelfand bosonic triple over $\mathcal{H}_{1}$

$$
\mathcal{B}_{1}^{\infty} \subset \mathcal{B}_{1}^{0} \subset \mathcal{B}_{1}^{-\infty}
$$

is transformed into the holomorphic Bargmann-Hida Gelfand super $*$-triple over $\mathcal{H}$

$$
\mathcal{B}^{\infty} \subset \mathcal{B}^{0} \subset \mathcal{B}^{-\infty}
$$




\subsection{Second quantization of classical super observables}

Following the chain rule, we define the odd and directional complex derivatives

$$
\partial_{z_{1}}=\partial_{\varpi z_{1}} \varpi^{\dagger}, \quad \partial_{z_{1}^{*}}=\partial_{z_{1}^{*}} \varpi^{\dagger}
$$

These analytic odd directional derivatives coincide with the left and right algebraic fermionic derivatives from [4].

Together with the even directional derivatives $\partial_{z_{0}}$ and $\partial_{z_{0}^{*}}$ they define the directional super derivatives $\partial_{z} \equiv \partial_{z_{0}}+\partial_{z_{1}}$ and $\partial_{z^{*}} \equiv \partial_{z_{0}^{*}}+\partial_{z_{1}^{*}}$. The super annihilation operators

$$
\hat{z}^{\dagger}: \mathcal{B}^{-\infty} \rightarrow \mathcal{B}^{-\infty} \text { if } z^{*} \in \mathcal{H}^{\infty}, \quad{\widehat{z^{*}}}^{\dagger}: \mathcal{B}^{\infty} \rightarrow \mathcal{B}^{\infty} \text { if } z \in \mathcal{H}^{-\infty}
$$

The corresponding super creation operators are the super adjoint multiplication operators $\hat{z}$ and $\widehat{z^{*}}$. The canonical super commutation relations: If $z^{*} \in \mathcal{H}^{\infty}, w \in \mathcal{H}^{-\infty}$, then

$$
\left[\widehat{z}^{*}, \hat{w}\right]=0=\left[\widehat{z}^{\dagger}, \hat{w}^{\dagger}\right], \quad\left[\widehat{z}^{\dagger}, \hat{y}\right]=z^{*} w
$$

As in the bosonic case, the classical super observables are analytic functionals $\Theta \in$ $\left(\mathcal{B} \otimes \mathcal{B}^{*}\right)^{-\infty}$.

The corresponding classical bosonic observable is the composition $\Theta^{\varpi} \equiv \Theta \circ \varpi^{\dagger}$. The normal bosonic quantum operator $\Theta^{\varpi}\left(\hat{z}, \widehat{z}^{\dagger}\right)$ has a unique super counterpart $\Theta\left(\hat{z}, \widehat{z^{*}}\right)$. Thus, any continuous linear operator in a Gelfand super triple has a unique normal bosonic symbol $\widetilde{\Theta}^{\varpi}\left(z^{*}, z\right)$ and the associated anti-normal symbol $\Theta^{\varpi}\left(z, z^{*}\right)$.

In particular, $\partial_{z}^{\varpi}, \partial_{z^{*}}^{\varpi}$ are the bosonic counterparts of the super directional derivatives, and $\hat{z}^{\varpi},{\widehat{z^{*}}}^{\varpi}$ of the super multiplication operators. Then $e^{{\widehat{z^{*}}}^{\dagger}} \varpi$ corresponds to $e^{{\widehat{z^{*}}}^{\dagger}}$, and $e^{\hat{z} \varpi}$ to $e^{\hat{z}}$. Thus

$$
\Theta\left(\hat{z},{\widehat{z^{*}}}^{\dagger}\right)^{\varpi}=\varpi^{\dagger} \Theta\left(\hat{z},{\widehat{z^{*}}}^{\dagger}\right) \varpi
$$

so that the matrix elements of the corresponding operators coincide: If $\Psi \in \mathcal{B}$, then

$$
\Psi^{*} \Theta\left(\hat{z}, \widehat{z}^{*}\right) \Psi=\Psi^{\varpi *} \Theta^{\varpi}\left(\hat{z}, \widehat{z}^{*}\right) \Psi^{\varpi}
$$

\section{Anti-Normal Super Feynman Integral}

Here we derive the anti-normal version of Feynman integral for the transformation matrix elements of Schrödinger super operators. In view of (3.14), we consider the bosonic case only.

Let $\left\{p_{n}\right\}$ be a flag of finite dimensional orthogonal projectors in $\mathcal{H}^{\infty}$ (i.e., an increasing sequence of projectors which are orthogonal in $\mathcal{H}$ and strongly converging to the unit operator in $\mathcal{H}^{\infty}$. They naturally define the flag of finite dimensional orthogonal projectors in the Gelfand triple $\mathbb{H}$, and therefore the corresponding flag of infinite dimensional orthogonal projectors $\hat{p}_{n}$ in the Gelfand triple $\mathbb{B}$.

Let $\widehat{H} \equiv \Theta\left(\widehat{z}, \widehat{z}^{*}\right)$. Assume that

$$
\Theta\left(z, z^{*}\right) \geq 0
$$


The contractions $\widehat{H}_{n} \equiv \hat{p}_{n} \Theta\left(\widehat{z}, \widehat{z}^{*}\right) \hat{p}_{n}$ are operators in the Bargmann-Hida triples $\mathcal{B}_{n} \equiv$ $\hat{p}_{n} \mathcal{B}$ over the finite-dimensional Hermitian spaces $\mathcal{H}_{n} \equiv p_{n} \mathcal{H}$.

Moreover, $\Theta\left(p_{n} z, p_{n} z^{*}\right)$ is the anti-normal symbol of $\widehat{H}_{n}$. (By $(2.27)$, this is straightforward for normal symbols, and then, by (2.33), for anti-normal as well.)

Note, we have identified the finite-dimensional spaces $\mathcal{H}_{n}$ with the Gelfand triples $\mathcal{H}_{n} \subset$ $\mathcal{H}_{n} \subset \mathcal{H}_{n}$. The Minlos Gauss measure is the standard Gauss measure on $\mathcal{H}_{n}$, so that $\mathcal{B}_{n}$ are (unbounded) operators on the Hilbert spaces $\mathcal{B}_{n}^{0}$ (see [5]) with the dense domains $\mathcal{B}_{n}^{\infty}$.

By $(4.1), \widehat{H}_{n}$ are positive definite symmetric operators on the Hilbert spaces $\mathcal{B}_{n}^{0}$ with the dense domains $\mathcal{B}_{n}^{\infty}$. They have Friedrichs self-adjoint extensions which are denoted again as $\widehat{H}_{n}$.

Now the transition amplitudes in $\mathcal{H}_{n}^{\infty}$ are

$$
\left\langle p_{n} z_{t} \mid \hat{p}_{n} z_{0}\right\rangle=e^{p_{n} z_{0} *} e^{-i t \widehat{H}_{n}} e^{p_{n} z_{0}}
$$

As in [16, pp. 69-70], consider the strongly differentiable family of operators $\widehat{A}_{n, \tau}, 0 \leq \tau \leq t$, in $\mathcal{B}$

$$
\left[\widehat{A}_{n, \tau} \Psi\right]\left(z_{0}^{*}\right)=\int d z^{*} d z e^{-z^{*} z} e^{z_{0}^{*} z} e^{-i \Theta_{n}\left(z^{*}, z\right) \tau} \Psi\left(z^{*}\right)
$$

Since $\left|e^{-i \Theta_{n}\left(z^{*}, z\right) t}\right|=1$, the operator norms are $\left\|\widehat{A}_{n, \tau}\right\| \leq 1$ in $\widehat{H}_{n}$.

Besides, the strong $t$-derivative $(d / d t) \widehat{A}_{n, \tau}(0)=\widehat{H}_{n}$ on the exponential states. Then, by the Chernoff's product theorem (see [8]), the evolution operator is

$$
e^{-i \hat{H}_{n}}=\lim _{N \rightarrow \infty}\left[\widehat{A}_{n, t / N}\right]^{N}
$$

Its kernel is the kernel contraction of the kernels of the factors

$$
\int \prod_{j=1}^{N} d z_{j}^{*} d z_{j} \exp \sum_{j=0}^{N}\left[\left(z_{j+1}-z_{j}\right)^{*} z_{j}-i t \Theta_{n}\left(z_{j}^{*}, z_{j}\right) / N\right]
$$

where $\left.z_{N+1}=z_{t}, z_{0}=z_{0}\right)$.

Thus, the amplitude $e^{p_{n} z_{t}^{*}} e^{-i t \widehat{H}_{n}} e^{p_{n} z_{0}}$ is the $N$-iterated Gaussian integral over $\mathcal{H}$ which, by the Fubini's theorem, is equal to the $N$-multiple Gaussian integral over $\mathcal{H}^{N}$.

In the notation $\tau_{j}=j t / N, z_{\tau_{j}}=z_{j}, j=0,1,2, \ldots, N$, and $\Delta \tau_{j}=\tau_{j+1}-\tau_{j}$, the multiple integral is

$$
\left.\int \prod_{j=1}^{N} d z_{\tau_{j}}^{*} d z_{\tau_{j}} \exp i \sum_{j=0}^{N} \Delta t_{j}\left[-i\left(\Delta z_{\tau_{j}} / \Delta \tau_{j}\right)^{*} z_{\tau_{j}}\right\rangle-\Theta_{n}\left(z_{\tau_{j}}^{*}, z_{\tau_{j}}\right)\right] .
$$

Its limit as $N \rightarrow \infty$ is a rigorous mathematical definition of the heuristic anti-normal Feynman integral

$$
\int_{z_{0}}^{z_{t}} \prod_{0<\tau<t} d z_{\tau}^{*} d z_{\tau} \exp \int_{0}^{t} d \tau\left[\left(\partial_{\tau} z_{\tau}^{*}\right) z_{\tau}-i \Theta_{n}\left(z_{\tau}^{*}, z_{\tau}\right)\right]
$$

over classical histories $z_{\tau}$ between $z_{0}$ and $z_{t}$ in $\mathcal{H}_{n}$. 
Since the quantum amplitude is

$$
\left\langle z_{t}^{*} \mid z_{0}\right\rangle=\lim _{n \rightarrow \infty}\left\langle p_{n} z_{t}^{*} \mid p_{n} z_{0}\right\rangle
$$

it is equal to the iterated limit of (4.5) as $N \rightarrow \infty$ is followed by the limit as $n \rightarrow \infty$. This iterated limit is a rigorous mathematical definition of the heuristic anti-normal Feynman integral for the amplitude $\left\langle z_{t} \mid z_{0}\right\rangle$

$$
\int_{z_{0}}^{z_{t}} \prod_{0<\tau<t} d z_{\tau}^{*} d z_{\tau} \exp \int_{0}^{t} d \tau\left[\left(\partial_{\tau} z_{\tau}^{*}\right) z_{\tau}-i \Theta\left(z_{\tau}^{*}, z_{\tau}\right)\right]
$$

over all classical histories $z_{\tau}$ between $z_{0}$ and $z_{t}$ in $\mathcal{H}^{\infty}$.

The non-negativity condition of the anti-normal symbol may be replaced just by its boundedness from below.

\section{References}

[1] G. Agarwal and E. Wolf, Calculus for functions of noncommuting operators and general phasespace methods in quantum mechanics, I, II, III, Phys. Rev. D2 (1970) 2161-2186, 2187-2205, $2206-2225$.

[2] J. C. Baez, I. E. Segal and Z. Zhou, Introduction to Algebraic and Constructive Quantum Field Theory (Princeton University Press, 1992).

[3] V. Bargmann, Remarks on a Hilbert space of analytic functions, in Proc. National Acad. Sci. USA, Vol. 48 (Washington D.C, 1962), pp. 199-204.

[4] F. A. Berezin, The Method of Second Quantization (Academic Press, 1966).

[5] F. A. Berezin, Wick and anti-wick Symbols of operators, Math. USSR Sbornik 15 (1971) 577606 .

[6] F. A. Berezin, Introduction to Superanalysis, Edited and with a foreword by A. A. Kirillov. With an appendix by V. I. Ogievetsky. Translated from the Russian by J. Niederle and R. Kotecký. Translation edited by D. Leites. Mathematical Physics and Applied Mathematics, 9. D. Reidel Publishing Co., Dordrecht.

[7] V. I. Bogachev, Gaussian Measures (American Mathematical Society, 1998).

[8] P. Chernoff, Note on product formulas for operator semigroups, J. Func. Analysis 2 (1968) 238-242.

[9] P. Dirac, Theory of the emission and absorption of radiation, in Proc. Royal Soc. A London, Vol. 144 (1927), pp. 234-262.

[10] P. Dirac, Lectures on Quantum Field Theory (Yeshiva University, N.Y., 1966).

[11] A. Dynin, Energy-mass spectrum of Yang-Mills bosons is infinite and discrete, arXiv:math$\mathrm{ph} / 09034727 \mathrm{v} 2$.

[12] V. Fock, Konfigurationsraum und zweite Quantulung, Zeitschrift für Physik 75 (1932) 622647. Also in: Selected Works, V. A. Fock: Quantum Mechanics and Quantum Field Theory (Chapman and Hall, 2004).

[13] V. Fock, Zur Quantumelektrodynamic, Phys. Zs. Sowjet 6 (1934) 425-469. Also in: Selected Works, V. A. Fock: Quantum Mechanics and Quantum Field Theory (Chapman and Hall, 2004).

[14] K. Friedrichs, Mathematical Aspects of the Quantum Theory of Fields (Interscience, 1953).

[15] I. Gelfand and N. Vilenkin, Generalized Functions, Vol. 4 (Academic Press, 1964).

[16] J. R. Klauder and B. Skagerstam, Coherent States (World Scientific, 1985).

[17] P. Kree, Calcule symbolique et second quantification des functions semiholomorphes, CR Acad. Sc. Paris, 284A (1974) 25-28.

[18] L. Landau and R. Peierls, Quantenelekrodynamik in Kofigurationsraum, Zeitschrift für Physik 62 (1930) 188. Collected Papers of L.D. Landau (Pergamon, 1967). 
[19] L. Landau and R. Peierls, Erweiterung des Unbestimmtheitsprinzips für die relativistische Quantentheorie, Zeitschrift für Physik, 69 (1931) 56. Collected papers of L.D. Landau (Pergamon, 1967).

[20] Yu. Manin, Gauge Field Theory and Complex Geometry, Vol. 289, 2nd edn. (Springer, Berlin, 1997).

[21] P.-A. Meyer, Quantum Probability for Probabilists, in Comprehensive Studies in Mathematics, in Lecture Notes in Mathematics, Vol. 1538 (Springer, 1995).

[22] R. Minlos, Felix Alexandrovich Berezin: A Brief Scientific Biography, in Topics in Statistical and Theoretical Physics. F.A. Berezin Memorial Volume, R. L. Dobrushin, R. A. Minlos, M. A. Shubin and A. M. Vershik (eds.): American Mathematical Society Translations, Series 2, 177. Advances in the Mathematical Sciences, 32 (American Mathematical Society, Providence, RI, 1996). Letters in Math. Physics 74(1) (2005); M. Shifman (ed.), Felix Berezin (World Scientific, 2007), p. 43.

[23] J. Moyal, Quantum mechanics as a statistical theory, in Proc. Camb. Phil. Soc., Vol. 45 (Cambridge, 1949), pp. 99-124.

[24] Yu. Neretin, Berezin's Method of Second Quantization. 40 Years After: in Recollections on Felix Alexandrovich Berezin - the Founder of Supermathematics, E. G. Karpel and R. A. Minlos (compilers), D. Leites and I. Tyutin (eds.), (MCCME, Moscow), pp. 382 (in Russian).

[25] J. von Neumann, Mathematical Foundations of Quantum Mechanics (Princeton University Press, 1955).

[26] N. Obata, White Noise Calculus and Fock Space, Lecture Notes in Mathematics, Vol. 1557 (Springer, 1994).

[27] A. Pietsch, Nuclear Locally Convex Spaces (Springer, 1972) (Translated from German).

[28] M. Reed and B. Simon, Methods of Modern Mathematical Physics, II (Academic Press, New York, 1972).

[29] H. Weyl, Theory of Groups and Quantum Mechanics (Dover, 1950).

[30] E. Wigner, On the quantum correction for thermodynamic equilibrium, Phys. Rev. 40 (1932) 749-759. 